\title{
Dolor neuropático en pacientes renales crónicos. Revisión de la literatura
}

\author{
S. Villate ${ }^{1}$, M. J. Ledesma y J. J. Martín ${ }^{1}$ \\ Servicios de ${ }^{1}$ Neurología ${ }^{2}$ Nefrología. Sanatorio Allende. Córdoba, Argentina
}

Villate S, Ledesma MJ, Martín JJ. Dolor neuropático en pacientes renales crónicos. Revisión de la literatura. Rev Soc Esp Dolor 2014; 21(3): 175-181.

\begin{abstract}
Pain is a common symptom in patients with chronic renal failure (CRF), being reported by $50 \%$ of such patients; the most common presentation is osteoarticular pain, followed by neuropathic pain, especially associated with polyneuropathy. Its approach, as well a sits complications are frequently a problem among physicians.

Pain is not an isolated symptom in these patients, they are usually exposed to stressful and emotional alterations such as depression or anxiety. Recent updates define functional chronic pain syndromes as those diseases characterized by daily pain and discomfort related to different parts of the body, common manifestations in these patients. Multimodal analgesia theory is a choice for pain management, here pain is part of complex syndrome and not the only symptom.

The aim of this study is to review therapeutic options for treating neuropathic pain in patients with chronic renal failure (CRF).
\end{abstract}

Key words: Chronic kidney disease. Neuropathic pain. Analgesia.

\section{RESUMEN}

El dolor es un síntoma frecuente en pacientes con insuficiencia renal crónica (IRC), siendo referido por el $50 \%$ de los

Recibido: 10-07-13.

Aceptado: 20-09-13. mismos en forma habitual; la presentación más prevalente es el dolor osteoarticular, seguido por el neuropático, especialmente asociado a polineuropatía. Su abordaje, como así también sus complicaciones, son motivo de preocupación frecuente en los médicos tratantes.

El dolor no es un síntoma aislado en dichos pacientes; habitualmente se encuentran expuestos a estresantes y presentan alteraciones emocionales como depresión o ansiedad. Actualizaciones recientes definen los síndromes funcionales dolorosos crónicos como aquellos cuadros clínicos caracterizados por dolor cotidiano y disconfort relacionado a diferentes partes del cuerpo; estas son manifestaciones habituales en estos pacientes. La teoría de analgesia multimodal plantea una opción para el manejo del dolor, en donde este es un síntoma dentro de un síndrome aún más complejo.

El objetivo del presente trabajo consiste en realizar una revisión acerca de las opciones terapéuticas para tratar el dolor neuropático en pacientes con insuficiencia renal crónica (IRC).

Palabras clave: Insuficiencia renal crónica. Dolor neuropático. Analgesia multimodal.

\section{INTRODUCCIÓN}

En Argentina la incidencia de pacientes en diálisis crónica ha tenido un crecimiento exponencial, llegando a 153 pacientes por millón de habitantes en el año 2010 (1). El dolor es un síntoma frecuente, reportado en hasta el 50$80 \%$ (2) de los pacientes en hemodiálisis, influyendo negativamente en su calidad de vida (3). La creciente población de pacientes con insuficiencia renal crónica sumada a la alta prevalencia del dolor como síntoma habitual nos plantea una problemática cotidiana y a su vez compleja (4).

Presentamos una actualización acerca de los principales síndromes dolorosos en el paciente renal crónico y en diálisis haciendo hincapié en el dolor neuropático y sus diferentes etiologías. Finalmente revisamos las opciones 
terapéuticas y aspectos generales del manejo farmacológico en esta población de pacientes.

\section{LA ENFERMEDAD RENAL CRÓNICA}

La enfermedad renal crónica (ERC) es un problema de salud global, con una incidencia creciente y que representa una importante carga económica para el sistema. Lamentablemente, aún hoy es subdiagnosticada y subtratada, perdiéndose oportunidades para la prevención de la progresión a enfermedad renal crónica terminal (5).

De acuerdo a las guías K/DOQI, la IRC se define como la disminución de la tasa de filtrado glomerular (TFG) o signos de daño renal que persisten por más de 3 meses. El valor normal del la TFG varía de acuerdo a edad, sexo y tamaño corporal. En adultos jóvenes es de 120 a $130 \mathrm{ml} / \mathrm{min}$ por $1,73 \mathrm{~m}^{2}$. Los marcadores de daño renal, aún en presencia de TFG normal son: proteinuria persistente (medida como un cociente albuminuria/creatininuria mayor a $30 \mathrm{mg} / \mathrm{g}$ en muestra aislada), anomalías en el sedimento urinario o en estudios por imágenes (5).

Según el valor de la TFG la insuficiencia renal crónica (IRC) se divide en estadios del 1 al 5, siendo este último el fallo renal que requiere terapia de reemplazo renal (diálisis o trasplante) (5) (Tabla I).

La TFG se estima a partir de los niveles de creatinina plasmática usando ecuaciones a partir de edad, sexo, raza y tamaño corporal (5).

Estas son:

Cockcroft-Gault:

Cl. creat $(\mathbf{m l} / \mathbf{m i n})=[(140-$ edad $) \times($ peso en $\mathrm{kg}) \times(0,85$ si mujer $)] /(72 x$ creatinina plasmática en $\mathrm{mg} / \mathrm{dl}$ )

MDRD abreviada:

$\boldsymbol{F G}\left(\mathbf{m l} / \mathbf{m i n} / \mathbf{1}, \mathbf{7 3} \mathbf{m}^{2}\right)=186 \times$ [creatinina plasmática $(\mathrm{mg} / \mathrm{dl})]-1,154 \times$ (edad)-0,203 $\times(0,742$ si mujer $) \times(1,21$ si raza negra)
Esta última tiene la ventaja de haber sido validada en un amplio grupo de pacientes con diversas patologías y comparada con mediciones directas con iotalamato.

En algunos casos se debe realizar una medición del clearence de creatinina en orina de 12 o $24 \mathrm{~h}$ : edades o tamaños corporales extremos, malnutrición u obesidad severas, enfermedades del musculoesquelético, para o cuadriplejía y/o vegetarianos (5).

\section{DOLOR EN PACIENTES CON IRC}

El dolor es una manifestación clínica prevalente en pacientes en IRC y más en aquellos que se encuentran en hemodiálisis (HD) (6).

La prevalencia de dolor en los pacientes en HD varía significativamente según las series estudiadas, desde un $37 \%$ hasta el $50 \%$ (7).

Un trabajo realizado en la Unidad de Hemodiálisis de Manacor, Mallorca, muestra que el 82,1\% de los pacientes afirmaron tener dolor y el $50 \%$ expresaron haber tenido una experiencia dolorosa en las últimas 24 horas. En la mayoría de los pacientes este fue de intensidad leve a moderada (2). En un estudio en una Unidad de Hemodiálisis en Canadá se realizó una encuesta en 205 pacientes, de los cuales el $50 \%$ refirió haber tenido alguna experiencia dolorosa. De estos, el $63 \%$ presentó dolor de tipo musculoesquelético, el 13,6\% relacionado al procedimiento dialítico y el $12,6 \%$ secundario a polineuropatía (8).

En forma coincidente, González describió que el dolor musculoesquelético fue el más frecuente $(60,31 \%)$, siendo principalmente atribuido a osteoartritis y osteoporosis $(23,50 \%)$. El dolor de causa neuropática representó el $12,69 \%$, la mitad secundaria al síndrome del túnel car-

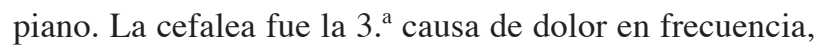
siendo referida por el 9,52\% de los pacientes (4). Este suele ser parte del llamado síndrome de desequilibrio, asociado a los rápidos cambios de osmolaridad en diálisis, o en otros casos expresión de alcalosis metabólica

TABLA I. ESTADIOS DE GRAVEDAD DE LA ENFERMEDAD RENAL CRÓNICA

\begin{tabular}{lll}
\hline Estadios & Descripción & TFG $\left(\mathrm{ml} / \mathrm{min} 1,73 \mathrm{~m}^{2}\right)$ \\
\hline 1 & Lesión renal con TFG normal o hiperfiltración & $>90$ \\
2 & Lesión renal con leve disminución de TFG & $60-89$ \\
3 & Moderada disminución de TFG & $30-59$ \\
4 & Avanzada disminución de TFG & $15-29$ \\
5 & Insuficiencia renal & $<15$ o diálisis \\
\hline
\end{tabular}

Los estadios 1 y 2: requieren la presencia de lesión renal (en sedimento, pruebas de imagen e histología). Modificada de cita 5. 
por concentraciones de bicarbonato elevadas en el baño de diálisis $(3,5)$.

La enfermedad vascular periférica representó el 7,93\% de las causas de dolor, principalmente presentada como claudicación intermitente (7). Otra forma de dolor relacionada a isquemia es el robo vascular asociado a las fístulas arteriovenosas en la circulación distal a la misma (8).

Con respecto al momento en que se presenta el dolor, se observó un claro aumento de la sensación de dolor en el día de la diálisis en un $71,4 \%$. Un 2,9\% refiere no tener dolor el día que no tienen diálisis, y el $25,7 \%$ no notan diferencia alguna entre los diferentes parámetros valorados. Cuando los pacientes no están en diálisis refieren también reagudizaciones del dolor sobre todo al realizar esfuerzo físico (7).

En el estudio de Davidson, ya mencionado, la única diferencia significativa entre los pacientes con y sin dolor es el tiempo de permanencia en HD (9). La severidad del dolor fue significativamente mayor en pacientes blancos e hipertensos (6).

Por su frecuencia y relación particular con la insuficiencia renal ampliaremos la polineuropatía urémica y diabética.

\section{Polineuropatía urémica}

El término uremia describe signos y síntomas que acompañan al fallo renal que no pueden ser explicadas por alteraciones en el volumen extracelular, concentraciones iónicas o en los niveles de productos de síntesis renal. Dentro de las múltiples manifestaciones de la uremia, una de las más frecuentes es la neuropatía urémica (10).

Esta es una polineuropatía sensitivo-motora simétrica distal que afecta en forma más frecuente los miembros inferiores (MMII). Característicamente progresa en el curso de meses. Se presenta usualmente cuando la TFG es menor a $12 \mathrm{ml} / \mathrm{min} / 1,73 \mathrm{~m} \mathrm{(10)}$.

A nivel ultraestructural se caracteriza por disminución del diámetro axonal, reorganización de la mielina y progresivamente degeneración completa del axón (10).

En cuanto a la fisiopatogenia hay varias teorías, algunas la relacionan a desmielinización secundaria de las columnas posteriores de la médula espinal (10). Fraser y Arieff postularon que las neurotoxinas deplecionan los suministros de energía del axón al alterar enzimas que participan en su producción. Además, las toxinas urémicas podrían ingresar al espacio endoneural y causar daño neuronal directo (11).

La paratohormona $(\mathrm{PTH})$ es una de las toxinas urémicas principales y una de las pocas en las que se ha demostrado relación causal, ya que esta aumenta los niveles intracelulares de calcio alterando la función axonal (10).

El hallazgo clínico más precoz es la pérdida del reflejo aquiliano. Los estudios de conducción nerviosa conti- núan siendo el gold standard diagnóstico, observándose neuropatía axonal, reducción de la amplitud sensorial con relativa conservación de la velocidad de conducción (10).

Las latencias distales prolongadas y la reducción de la amplitud del potencial de acción son causadas por la disminución en la densidad de las fibras sensitivo-motoras largas. La amplitud sensorial a nivel del nervio sural es el indicador más sensible de neuropatía urémica, ya que está presente en el $50 \%$ de los casos. Otros parámetros considerados sensibles son anomalías en las respuestas tardías de las ondas $\mathrm{F}$ y reflejos $\mathrm{H}$, el umbral de detección de la vibración en el pie y la disminución de la velocidad de conducción nerviosa (12).

\section{Neuropatía diabética}

La neuropatía diabética es una complicación común de la enfermedad asociada a alta morbilidad, deterioro de la calidad de vida y alto riesgo de amputaciones. En forma similar, la neuropatía autonómica cardiaca se asocia con complicaciones como isquemia coronaria silente, arritmias y alta mortalidad (13).

La fisiopatogenia es compleja, siendo secundaria a la interacción de los niveles de hiperglucemia, el metabolismo de la glucosa a través de las vías de los polioles, la vía de la hexosamina, activación inapropiada de proteínakinasa $\mathrm{C}$, disfunción de la bomba de $\mathrm{Na} / \mathrm{K}$ y acumulación de productos de glicosilación avanzada (13) (Fig. 1).

Clínicamente se presenta como una polineuropatía (PNP) distal simétrica a predominio de MMII con síntomas y signos de compromiso de fibras pequeñas y un dolor de tipo dolor urente. Para evaluar este tipo de fibras se utiliza un monofilamento, un alfiler y algodón en el dorso del $1{ }^{\text {er }}$ y $2 .^{\circ}$ dedo del pie o en la superficie plantar. La función de fibras mayores se evalúa con un diapasón de 256-Hz o movimientos de los dedos para evaluar propiocepción (13).

\begin{tabular}{|c|c|}
\hline \multirow{2}{*}{$\begin{array}{l}\text { Primer escalón de analgesia de la OMS } \\
\text { (AINE) }\end{array}$} & Paracetamol \\
\hline & \\
\hline \multirow{3}{*}{$\begin{array}{c}\text { Segundo escalón de analgesia de la } \\
\text { OMS } \\
\text { (opioides menores) } \\
+ \\
\text { adyuvantes (gabapentina, pregabalina) }\end{array}$} & \\
\hline & Tramadol \\
\hline & Code \\
\hline \multirow{3}{*}{$\begin{array}{l}\text { Tercer escalón analgesia de la OMS } \\
\text { (opioides mayores) } \\
+ \\
\text { Adyuvantes (gabapentina, pregabalina) }\end{array}$} & \\
\hline & Oxicodona \\
\hline & Morfina \\
\hline
\end{tabular}

Fig. 1. Esquema analgésico propuesto por la OMS (20). 
Rev. Soc. Esp. del Dolor, Vol. 21, N. ${ }^{\circ}$ 3, Mayo-Junio 2014

\section{TRASTORNOS DE ESTADO DE ÁNIMO DEL PACIENTE INSUFICIENTE RENAL}

$\mathrm{Al}$ evaluar un síntoma tan subjetivo como el dolor es importante recordar la presencia de diversos déficits neuropsicológicos en pacientes con trastorno renal crónico. El proceso de depuración de la sangre durante la diálisis se asocia a una normalización de la actividad neuronal y una mejora en el rendimiento cognitivo en diversas funciones neuropsicológicas. En este sentido, la diálisis podría favorecer un mejor funcionamiento de los procesos perceptivos y psicofisiológicos implicados en la inhibición aferente del dolor mediada por el reflejo barorreceptor (6).

Debemos tener en cuenta también los factores asociados al fenómeno de la analgesia inducida por estrés. El procedimiento de diálisis se inicia en un contexto de relativo estrés; enfermeras, aparatos, tubos, olores, agujas, quitarse la ropa, fenómeno de la bata blanca, etc. Entre los elementos más estresantes del procedimiento se encuentra la conexión de las agujas a la fístula arteriovenosa. Además del dolor producido por la punción de las dos agujas, los pacientes suelen preocuparse por posibles problemas en la fístula $(7,9)$.

La depresión es un problema psicológico de alta prevalencia (10-66 \%) en pacientes con IRC y puede ser abordado con intervenciones psicológicas (IP) y/o combinadas (9). En escenarios concretos, como pueden ser las unidades de diálisis peritoneal, la depresión está infradiagnosticada y es de difícil tratamiento. Hay pocos estudios controlados y mucho menos aleatorizados que aborden este problema (14).

La tasa de prevalencia de trastornos de ansiedad en pacientes en hemodiálisis se sitúa cerca del $45,7 \%$ y afectan negativamente a la calidad de vida. Según Cukory cols., estos pacientes se enfrentan a un alto número de estresares (14). Estos pacientes se enfrentan a un alto número de estresares como son la restricción hídrica, dieta, insomnio, pérdida de rol social y limitaciones sociales.

\section{CONSIDERACIONES TERAPÉUTICAS}

Los pacientes insuficientes renales que se encuentran en HD presentan múltiples comorbilidades (14). Al evaluar a un individuo con dichas características en un consultorio de dolor es necesario contextualizar el caso para poder emprender la terapéutica apropiada.

La insuficiencia renal (IR) altera con frecuencia el volumen de distribución del fármaco. Así mismo, también es posible una disminución de la capacidad de fijación tisular en lugar de la unión a las proteínas plasmáticas. Este fenómeno reduce el contenido corporal de fármaco a cualquier nivel plasmático y el volumen de distribución. Una dosis estándar provoca un mayor nivel plasmático y mayor in- tensidad de efecto en un paciente urémico en comparación con una persona sin IR (15).

La vida media de los fármacos en un paciente insuficiente renal aumenta lentamente hasta que el aclaramiento de creatinina disminuye por debajo de $30 \mathrm{ml} / \mathrm{min}$ (15).

Sabiendo la TFG, en base al cálculo estimado por las fórmulas antes descriptas, podremos adaptar la dosis de los fármacos a utilizar (16) (Tabla II).

Es importante destacar la dosificación justa que requieren los pacientes con IRC, distinto de aquel que se encuentran en HD. En esta última instancia los fármacos pueden ser retirados con el proceso de diálisis, debido a su unión o no a proteína, y el volumen de distribución se modifica (15).

La dosis inicial administrada a un paciente con IR es la misma que la de un paciente con función renal normal, a menos que existan factores hemodinámicos de depleción de volumen, en cuyo caso se disminuye la dosis de carga al $75 \%$ (15).

La dosis de mantenimiento de muchos fármacos debe modificarse en pacientes con IR. Se utilizan dos métodos: alargar el intervalo entre dosis o disminuir la dosis. En la práctica, sin embargo, es muy común utilizar una combinación de ambos métodos (15). Tanto las estrategias de dosificación como los ajustes requeridos luego del proceso de HD se aprecian en la tabla II (16).

La concentración de amonio gástrico aumenta y amortigua así la acidez del estómago necesaria para intervenir en la absorción de determinados fármacos. También la neuropatía del sistema autónomo en pacientes diabéticos puede alterar los mecanismos absorción (15).

Según una revisión de los analgésicos más utilizados en pacientes con IRC, son en su mayoría antiinflamatorios no esteroideos (AINE), paracetamol, e inhibidores de la ciclooxigenasa 2 (COX2); entre los opiáceos usados encontramos morfina, propoxifeno y oxicodona. Las combinaciones más frecuentes halladas en la bibliografía indican el uso de propoxifeno con paracetamol, también morfina y un COX2 o morfina y AINE (17).

El tratamiento del dolor multimodal no es más que la combinación de dos o más fármacos y/o métodos analgésicos con el fin de potenciar la analgesia y disminuir los efectos colaterales. Se incorpora el concepto de fármacos adyuvantes, es decir, cualquier droga cuya indicación primaria no es para el tratamiento del dolor, pero que posee propiedades analgésicas en algunos síndromes dolorosos (18). En cuanto a los niveles de analgesia en pacientes con IRC la OMS establece el siguiente esquema analgésico (19).

En el primer nivel se encuentra el paracetamol; su estrategia de dosificación consiste en incrementar el intervalo de dosis a ocho en vez de seis horas cuando la TFG es menor a $10 \mathrm{ml} / \mathrm{h}(19)$.

La pregabalina es un fármaco del grupo de los neuromoduladores utilizado como adyuvante en el tratamien- 
TABLA II. DOSIFICACIÓN DE FÁRMACOS EN PACIENTES INSUFICIENTES RENALES

\begin{tabular}{|c|c|c|c|c|c|c|}
\hline \multirow[b]{2}{*}{ Fármaco } & \multirow{2}{*}{$\begin{array}{c}\text { Dosis con función renal } \\
\text { normal }\end{array}$} & \multirow[b]{2}{*}{ Método } & \multicolumn{3}{|c|}{ Ajuste para insuficiencia renal } & \multirow{2}{*}{$\begin{array}{c}\text { Suplemento después } \\
\text { de hemodiálisis }\end{array}$} \\
\hline & & & $\begin{array}{l}100-50 \\
\mathrm{ml} / \mathrm{min}\end{array}$ & $\begin{array}{l}50-10 \mathrm{mll} \\
\quad \mathrm{min}\end{array}$ & $\begin{array}{l}\text { Menos de } \\
10 \mathrm{ml} / \mathrm{min}\end{array}$ & \\
\hline Ibuprofeno & $1.200-1.800 \mathrm{mg} / \mathrm{día}$ & D & $100 \%$ & $75-50 \%$ & Evitar & No \\
\hline Naproxeno & $1 \mathrm{~g} /$ día & $\mathrm{D}$ & $100 \%$ & $75-50 \%$ & Evitar & No \\
\hline Diclofenaco & 100-150 mg/día & $\mathrm{D}$ & $100 \%$ & $75-50 \%$ & Evitar & No \\
\hline Piroxicam & 20 mg/día & $\mathrm{D}$ & $100 \%$ & $75-50 \%$ & Evitar & No \\
\hline $\begin{array}{l}\text { Clonixinato de } \\
\text { lisina }\end{array}$ & $125-250 \mathrm{mgc} / 6 \mathrm{~h}$ & $\mathrm{D}$ & $100 \%$ & $75 \%$ & $50 \%$ & No \\
\hline Celecoxib & 200 mg/día & $\mathrm{D}$ & $100-75 \%$ & $75-25 \%$ & Evitar & No \\
\hline Amitriptilina & 30-300 mg/día & $\mathrm{D}$ & $100 \%$ & $100 \%$ & $100 \%$ & No \\
\hline Clomipramina & 30-250 mg/día & $\mathrm{D}$ & \multicolumn{3}{|c|}{ No hay datos suficientes } & \\
\hline Nortriptilina & 20/200 mg/día & $\mathrm{D}$ & $100 \%$ & $100 \%$ & $100 \%$ & No \\
\hline Citalopram & 20 mg/día & $\mathrm{D}$ & $100 \%$ & $100 \%$ & Evitar & No \\
\hline Escitalopram & 10-20 mg/día & $\mathrm{D}$ & $100 \%$ & $100 \%$ & $50 \%$ & No \\
\hline Fluoxetina & 20 mg/día & $\mathrm{D}$ & $100 \%$ & $100 \%$ & $100 \%$ & No \\
\hline Paroxetina & 20-50 mg/ día & $\mathrm{D}$ & $100 \%$ & $75-50 \%$ & $50 \%$ & No hay datos \\
\hline Sertralina & $50-200$ mg/día & $\mathrm{D}$ & \multicolumn{3}{|c|}{ No hay datos suficientes } & \\
\hline Venlafaxina & 75-375 mg/día & $\mathrm{D}$ & $75 \%$ & $50 \%$ & $50 \%$ & No \\
\hline Codeína & $30 \mathrm{mg} / 4-6 \mathrm{~h}$ & $\mathrm{D}$ & $100 \%$ & $75 \%$ & $50 \%$ & No \\
\hline Paracetamol & $0,5-1 \mathrm{~g} / 4-6 \mathrm{~h}$ & I & $100 \%$ & Cada 6-8 h & Cada $8-12$ h & No \\
\hline Tramadol & $50-100 \mathrm{mg} / 6-8 \mathrm{~h}$ & I & Cada $8 \mathrm{~h}$ & Cada 12 h & Evitar & No \\
\hline Morfina & $10 \mathrm{md} / 4 \mathrm{~h}$ (titular dosis) & $\mathrm{D}$ & $100 \%$ & $75 \%$ & $50 \%$ & No \\
\hline Buprenorfina & $0,2-0,4 / 6-8 \mathrm{~h}$ & $\mathrm{D}$ & $100 \%$ & $100 \%$ & $100 \%$ & No \\
\hline Meperidina & $1-1,5 \mathrm{mg} / \mathrm{kg} / 3-4 \mathrm{~h}$ & $\mathrm{D}$ & $100 \%$ & $75 \%$ & $50 \%$ & No \\
\hline Oxicodona & $10 \mathrm{mg} / 12 \mathrm{~h}$ (titular dosis) & $\mathrm{D}$ & \multicolumn{4}{|c|}{ Iniciar con menos del $50 \%$ de dosis y titular } \\
\hline Fentanilo & $200 \mathrm{mg} /$ día y titular & $\mathrm{D}$ & $100 \%$ & $75 \%$ & $50 \%$ & No hay datos \\
\hline
\end{tabular}

Eliminación predominante: H: hepático; R: renal. Método de ajuste de dosis: I: intervalo de dosis; D: reducción de dosis. Modificada de cita 16.

to del dolor al bloquear el canal de calcio regulado por voltaje tipo $\mathrm{L}$ disminuyendo así el ingreso del mismo a la célula (19). En estudios controlados con placebo 300$600 \mathrm{mg}$ al día de pregabalina alivian significativamente el dolor. Los efectos adversos son mareos, ataxia, sedación, euforia y edema maleolar. En pacientes con TFG entre 30$60 \mathrm{ml} / \mathrm{min}$ se debe disminuir la dosis al $50 \%$ y luego se disminuye $50 \%$ más por cada caída del $50 \%$ de la TFG. Se elimina el $50 \%$ de una dosis durante una diálisis de $4 \mathrm{~h}$ por lo que requiere dosis suplementaria postdiálisis (3).

Gabapentina actúa de la misma manera, y las dosis requeridas son de $2.400 \mathrm{mg} / \mathrm{d}$. Sin embargo sólo se eli- mina por riñón y no se une a proteínas plasmáticas, por lo que la dosis se disminuye a $1.400 \mathrm{mg} / \mathrm{d}$ en clearence de creatinina (CCr) de 30-60 ml/min, $700 \mathrm{mg} / \mathrm{d}$ para $\mathrm{CCr}$ de $16-29 \mathrm{ml} / \mathrm{min}$ y $300 \mathrm{mg} / \mathrm{d}$ con CCr de $15 \mathrm{ml} / \mathrm{min}$. Los pacientes en diálisis deben recibir una dosis inicial de 300$400 \mathrm{mg}$ y luego 200-300 mg postdiálisis (13).

El tramadol es de elección en el segundo escalón. Su dosificación consiste en el aumento del intervalo cada doce horas y dosis menores $(50 \mathrm{mg})$; deben evitarse las fórmulas retardadas (12). La dosis máxima es de $200 \mathrm{mg} / \mathrm{d}$. Sólo el $7 \%$ de la dosis es removido en diálisis por lo que no requiere dosis de refuerzo (13). 
Rev. Soc. Esp. del Dolor, Vol. 21, N. ${ }^{\circ}$ 3, Mayo-Junio 2014

La buprenorfina se metaboliza en hígado; la droga indemne se elimina por vía biliar y los metabolitos por vía renal. Estos aumentaban en pacientes con IRC durante la infusión intravenosa de buprenorfina, hasta el momento sin reportes de efectos indeseados. Uno de sus metabolitos (B3G) mostró tener mayor índice de complicaciones al causar depresión respiratoria en animales de experimentación (19).

La morfina es metabolizada en el hígado generando morfina-3-glucorónido y morfina-6-glucorónido, este último acumulable en pacientes con IRC. Actualmente se considera la implementación de otros opioides antes de incorporar morfina y diamorfina al esquema analgésico (19).

La metadona se metaboliza en hígado, su principal metabolito se excreta por materia fecal y parte del fármaco intacto se elimina también por vía renal. La metadona ha cobrado relevancia al momento de seleccionar un opioide principalmente por sus características farmacodinámicas, sumada a las limitaciones de otros opioides. Aún así, debido a la toxicidad, riesgo de acumulación y variabilidad interindividual requiere la supervisión de médicos expertos al momento de implementarla en pacientes renales crónicos. Las pautas de titulación habituales se reducirán al 50 o $75 \%$ de las dosis recomendadas (19).

No hay consenso acerca del uso de oxicodona en pacientes con IRC; se metaboliza en hígado a dos productos activos, cuya eliminación se prolonga en la IRC (19).

Entre las opciones farmacológicas del tercer escalón se encuentra el fentanilo principalmente porque sus metabolitos son inactivos y no tóxicos. En cuanto a su administración se recomienda reducir la dosis conforme empeora la función renal, a un $75 \%$ si el aclaramiento de creatinina está entre 10 y $50 \mathrm{ml} / \mathrm{min}$ y al $50 \%$ si es menor a $10 \mathrm{ml} / \mathrm{min}$; los bolos no requieren ajuste de dosis. Por vía subcutánea se puede empezar con 25 microgramos y convertir luego a infusión continua, aunque hay pocos estudios que avalen su uso en infusión continua. La vía transdérmica, al igual que en pacientes sin insuficiencia renal, no es apropiada para el dolor incontrolado, por el largo tiempo requerido para ajustar la dosis. Su introducción es apropiada si el dolor está controlado por otra vía. Se han reportado casos de depresión respiratoria con parches de 25 microgramos en pacientes que no han usado opioides antes (19).

Los antidepresivos han sido utilizados como fármacos de primera línea en el tratamiento de diferentes cuadros de dolor neuropático (21). Aumentan la transmisión de noradrenalina y serotonina, en el cerebro y la médula espinal, mediante el bloqueo de su recaptación, por lo que se piensa que sus efectos analgésicos son independientes de los efectos antidepresivos centrales. En este sentido, duloxetina y amitriptilina inhiben la recaptación noradrenalina/ serotonina en una proporción similar (22). La severidad de síntomas dolorosos se vio disminuida en pacientes con depresión, fibromialgia y ha sido eficaz en el tratamiento del dolor neuropático periférico diabético. La duloxetina presenta menor incidencia de efectos adversos y su efecto terapéutico es precoz al compararla con amitriptilina $(23,24)$. La dosis habitual para amitriptilina es de 10$25 \mathrm{mg}$ antes de acostarse, aumentándola según tolerancia a 100-150 mg en una toma (3).

Ambos fármacos fueron eficaces en el tratamiento del dolor neuropático. Duloxetina, sin embargo, presentó como ventajas, un menor tiempo de inicio del efecto analgésico y una menor incidencia de efectos indeseables, que se minimizaron, significativamente, con dosis iniciales de $30 \mathrm{mg}$. El perfil de los efectos secundarios, asociados a duloxetina, fue ventajoso en relación al tipo de pacientes, con edad avanzada y patología previa (24). En pacientes con IRC la dosis máxima es de $60 \mathrm{mg} /$ día y no se recomienda con $\mathrm{TFG}<30 \mathrm{ml} / \mathrm{min}(5)$.

\section{CONSIDERACIONES FINALES}

Los síndromes funcionales dolorosos crónicos son aquellos cuadros clínicos caracterizados por dolor cotidiano y disconfort relacionado a diferentes partes del cuerpo (25). Generalmente hay ausencia de alteraciones estructurales, inflamatorias o bioquímicas que puedan explicar completamente los síntomas, y los pacientes padecen un severo deterioro de su calidad de vida debido a tratamientos poco efectivos (26). La prevalencia de los síndromes funcionales es de $15 \%$ de la población mundial y representa 20 al $50 \%$ de los motivos de consulta en atención primaria. Con frecuencia estos pacientes han sido vistos por múltiples especialistas y refieren falta de mejoría con los esquemas analgésicos administrados (26,27). Entre los síntomas dolorosos presentados se encuentra cefalea, dolor lumbar o cervical; pueden asociarse con insomnio, fatiga diaria, síntomas de colon irritables, disfunción temporomandibular y depresión (28).

El paciente en IRC presenta características propias del síndrome funcional doloroso y debe ser tratado de forma integral, no abordando el dolor únicamente. La teoría de analgesia multimodal establece el uso conjunto de fármacos para favorecer el efecto analgésico a dosis menores (18). Dentro de los adyuvantes, los antidepresivos duales como duloxetina o venlafaxina, parecerían ser más eficaces no sólo por disminuir y prevenir el dolor sino también por su efecto intrínseco antidepresivo, beneficioso en síndromes funcionales dolorosos.

A diferencia de lo establecido en la escalera analgésica de la OMS, creemos que en esta clase de pacientes deberían incorporarse esquemas terapéuticos que consideren el uso de paracetamol, junto con pregabalina y duloxetina en fases tempranas debido a su antecedente de dolor crónico 
y estado anímico. De ser necesario podrían seguir combinándose drogas como baclofeno, corticoides y morfina.

Finalmente no debemos olvidar que parte del tratamiento del dolor crónico se centra en los recursos no farmacológicos.

\section{CORRESPONDENCIA:}

Sebastian Villate

Servicio de Neurología

Sanatorio Allende

Córdoba, Argentina

e-mail: sebastianvillate@hotmail.com

\section{BIBLIOGRAFÍA}

1. Registro Argentino de Diálisis Crónica SAN-INCUCAI 2009-2010. Informe 2011. http://www.nefrodial.org.ar/ index.php

2. Rodríguez Calero M, Hernández Sánchez D. Unidad de Hemodiálisis. Evaluación del dolor crónico en una población de pacientes hemodializados. Fundación Hospital Manacor, Mallorca. Rev Soc Esp Enferm Nefrol 2007;10(2):137-43.

3. Daugirdas JT. Manual de diálisis. $4^{\circ}$ edición en español. Ed Wolters Kluwer Health España, 2008;2:34-56.

4. González C, Pizarro C. Dolor crónico en hemodializados: prevalencia, severidad y estrategias de afrontamiento. Revista El Dolor 2009;5119-25.

5. Andrew S, Levey MD. National Kidney Foundation Practice Guidelines for Chronic Kidney. Disease: Evaluation, classification, and stratification. Ann Intern Med 2003;139:137-47.

6. Pastor Armendariz M, Martínez Terceño M. Valoración del dolor en los pacientes en hemodiálisis. Rev Soc Esp Enferm Nefrol Madrid 2010;13(4).

7. Reyes del Paso G, Perales Montilla C. El curso de la hemodiálisis está asociado a cambios en el umbral de dolor y en las relaciones entre presión arterial y dolor. Nefrologia 2011;31(6):738-42.

8. Davison SN. Pain in hemodialysis patients: Prevalence, cause, severity, and management. Am J Kidney Dis 2003;42:1239-47.

9. Cerezo S, Barceló P. Dolor en hemodiálisis. DYT 2005;26(2):47-60.

10. Vagner Ramírez B, Bustamante Gómez PA. Uraemic neuropathy: A review. International Journal of Genetics and Molecular Biology 2012;3(11):155-60.

11. González O, González E. Fisiopatología del dolor. Artículo revisión. Rev Ven Anest 1998;3:1:26-33.
12. Ropper A, Adams SM, Victor. Principios de neurología. $9^{\text {a }}$ ed. 2011;45:1231-45.

13. Rodica Pop-Busui, Roberts L, Subramaniam P. The management of diabetic neuropathy in CKD. American Journal of Kidney Diseases 2010;55(2):365-85.

14. Leiva-Santos J, Sánchez-Hernández R. Cuidados de soporte renal y cuidados paliativos renales: revisión y propuesta en terapia renal sustitutiva. Nefrologia 2012;32(1):20-7.

15. Ortiz Sánchez Y, García Tasé M. Utilización de fármacos en insuficiencia renal. Rev Cubana Farm 2007;41(2).

16. Álvarez de Lara MA. Ajuste de Fármacos en la Insuficiencia Renal. En: Nefrología digital, sitio de la Sociedad Española de Nefrología. Disponible en: http://nefrologiadigital.revistanefrologia.com

17. Bailie G, Mason N. Analgesic prescription patterns among hemodialysis patients in the DOPPS: Potential for under prescription. Kidney International 2004;65:2419-25.

18. González de Meja, N. Analgesia multimodal postoperatoria. Rev Soc Esp Dolor 2005;12:112-8.

19. Murtagh F, Chai M. The use of opioid analgesia in end-stage renal disease patients managed without dialysis: Recommendations for practice. J Pain Palliat Care Pharmacother 2007;21(2):5-16.

20. Algoritmos en nefrología. Enfermedad renal crónica. Sociedad Española de Nefrología. 2012. Disponible en: http:// www.revistanefrologia.com/modules.php?name=editorial

21. Goldstein DJ, Lu Y, Detke MJ. Duloxetine vs. placebo in patients with painful diabetic neuropathy. Pain 2005;116:109-18.

22. Brannan SK, Mallinckrodt CH, Brown EB, et al. duloxetine $60 \mathrm{mg}$ once-daily in the treatment of painful physical symptoms in patients with major depressive disorder. J Psychiatr Res 2005;39:43-53.

23. Antidepressants for neuropathic pain. Cochrane Database Syst Rev 2005.

24. Finnerupa NB, Otto M. Algorithm for neuropathic pain treatment: An evidence based proposal. Pain 2005;118 (3):289-305.

25. Mayer E, Bushnell M. Functional pain syndromes: Presentation and pathophysiology. IASP Press 2009;1,2,3:25-176.

26. Clauw DJ, Chrousos GP. Chronic pain and fatigue syndromes: Overlapping clinical and neuroendocrine feautures and potential pathogenic mechanisms. Neuroimmunomodulation 1997;4:134-53.

27. Goldenberg DL, Clauw DJ, Fitzcharles MA.New Concepts in Pain Research and Pain Management of the Rheumatic Diseases. Semin Arthritis Rheum. 2011 Dec;41(3):319-34.

28. Jason LA, Taylor RR, Kennedy BA. Chronic fatigue syndrome, fibromyalgia, and multiple chemical sensitivities in a community-based sample of persons with chronic fatigue syndrome-like symptoms. Psychosomatic Medicine 2000;62:655-63. 